\title{
Acetaminophen Use in Pregnant Women and Their Neonates: Safe or Unsafe till Proven Otherwise?
}

\author{
John N. van den Anker ${ }^{a, b} \quad$ Karel Allegaert ${ }^{c-e}$

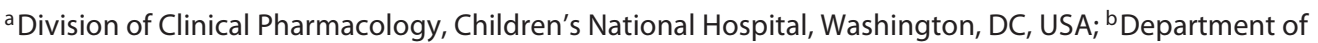 \\ Pediatric Pharmacology and Pharmacometrics, University of Basel Children's Hospital, Basel, Switzerland; \\ 'Department of Development and Regeneration, KU Leuven, Leuven, Belgium; ' Department of Pharmaceutical \\ and Pharmacological Sciences, KU Leuven, Leuven, Belgium; ' Department of Clinical Pharmacy, Erasmus MC, \\ Rotterdam, The Netherlands
}

In his commentary, Dr. Ola Saugstad tries to convince the readers of the journal that the replacement of acetylsalicylic acid with acetaminophen in the early 1980s has resulted in a sharp increase in autism spectrum disorder (ASD) in the USA. As a consequence, he warns against the use of prenatal and postnatal acetaminophen in pregnant women and their fetuses/neonates until we know more about a possible time period during the fetal/neonatal development where the developing brain might be more sensitive to potential side effects of acetaminophen and its metabolites [1]. He furthermore states that more care is needed when administering this "potentially toxic substance to individuals with developing brains." He finishes his commentary by advocating for proper follow-up if using acetaminophen in newborns and infants to detect potential long-term adverse effects.

We would like to discuss in this commentary the arguments Dr. Saugstad has used to reach the aforementioned conclusions and warnings, and at the end we would like to share with you our overall thoughts on this important topic.

Forty years ago, a link between the use of acetylsalicylic acid and Reye's syndrome [2] resulted in a substitution across the globe of acetylsalicylic acid with acetamin-

\section{KARGER}

(c) 2020 S. Karger AG, Basel

karger@karger.com

www.karger.com/neo ophen or ibuprofen. Some countries decided in favor of acetaminophen as replacement of acetylsalicylic acid whereas others preferred ibuprofen. There was not much rationale behind the choice of different countries to switch to either acetaminophen or ibuprofen.

Therefore, the fact that from 1970 to 2005 a sharp increase in autism in the USA was observed does not automatically seem to be linked to an increased use of acetaminophen as a replacement of acetylsalicylic acid worldwide because clearly there was an equal division between countries who switched to acetaminophen versus ibuprofen. Moreover, in the USA, the predominant switch was from acetylsalicylic acid to ibuprofen, whereas in many European countries acetaminophen became the preferred drug.

ASD is a neurodevelopmental disorder characterized by varying deficits in social interactions, disordered communication, and repetitive behavior patterns [3]. Signs that a child has autism are present in the early developmental stages and the symptoms cause significant impairment in many areas of functioning, including social, educational/occupational, and performance of everyday activities. It has been shown that more than half of ASD cases are attributable to environmental factors. The use of 
medications, cigarettes, later gestational age at the start of prenatal vitamins have all been correlated with ASD diagnosis [4]. Consequently, the search for additional environmental factors is very reasonable, but what about the potential mechanisms between exposure to acetaminophen and the development of ASD?

Ten years ago, Schultz hypothesized that disruption of the endocannabinoid system by acetaminophen might result in an increase in ASD, particularly in children with a decreased capacity to metabolize acetaminophen [5]. This hypothesis is further supported by animal experimental observations. Paracetamol drug metabolism results in p-aminophenol, a metabolite that interacts with cannabinoid receptors. Related to this, paracetamol and $\Delta(9)$-tetrahydrocannabinol, but not ibuprofen, resulted in developmental neurotoxicity in a juvenile mice model [6-8]. Furthermore, co-administration of a cannabinoid receptor agonist enhanced the developmental neurotoxicity of paracetamol [7].

The bulk of data in the human setting are observational, based on epidemiological linkage between exposure and outcome, by definition hampered by the association between exposure and indication (acetaminophen intake because of pain or fever). Masarwa et al. [9] recently reported in a systematic review on the link between prenatal paracetamol exposure and the risk for ADHD, ASD and hyperactivity, and suggested a pooled risk ratio of 1.34 (95\% CI 1.21-1.47), 1.19 (1.14-1.25) and 1.24 (1.041.43), respectively, but acknowledged that these observational data remain susceptible to several sources of bias. This paper in the American Journal of Epidemiology resulted in a fierce discussion between the authors of that paper and Damkier [10] underscoring that the issue of acetaminophen during pregnancy and childhood neurodevelopment remains controversial and contested [11, 12]. Masarwa and colleagues, in their response to the letter to the editor of Damkier, wrote: "We share Damkier's skepticism of the association between prenatal exposure to acetaminophen and neurodevelopmental outcomes, as well as his clinical concerns." The latter part of this response had to do with the fact that Masarwa et al. prematurely mentioned "recent alarming evidence on the teratogenicity of acetaminophen" in the same paper. The fact that this statement had no reference and was not supported by any evidence in their paper resulted in an exceptional and unnecessarily opinionated statement with substantial implications for health care professionals and pregnant women.

A very recent paper, published on January 1,2020, suggests the possibility that postnatal acetaminophen, mea- sured in doses consumed before age 2, may be a significant contributor to the risk of ASD among males in the US [13]. However, the real impact is unclear because other correlated variables were not included in the analysis. This paper also highlights the fact that we do not know much about the dose-concentration-effect relationship between exposure to acetaminophen and potential risk for ASD.

This automatically leads us to the paper of Ji et al. [14] who showed that kids with ASD as well as ADHD had higher concentrations of acetaminophen and its metabolites in cord blood. The question is of course if just measuring acetaminophen and its metabolites in cord blood and linking it to ASD/ADHD will help us explain the mechanism behind these findings. Is it the higher exposure to acetaminophen and its metabolites that "causes" ASD/ADHD or is the fact that you are going to develop ASD/ADHD the reason why you have a different clearance of acetaminophen? There is some evidence that supports the fact that kids with ASD/ADHD indeed have a different metabolism of acetaminophen and perhaps, as a consequence, have an elevated risk of adverse events if exposed to acetaminophen [15]. Furthermore, this design is also hampered because it was confounded by indication (maternal pain or fever during labor).

We would like to finish our commentary by applauding Dr. Saugstad for his provocative commentary that further underscores that we need to study childhood neurodevelopment following in utero exposure to drugs [1]. However, the issue of acetaminophen use during pregnancy and childhood neurodevelopment remains controversial and contested $[11,12]$. A recent study by Ystrom et al. [16] reported comparable weak signals for the risk of ADHD following maternal in utero exposure and paternal acetaminophen exposure prior to conception. This very important point should force the epidemiologically minded community to revisit the issue of causality and confounding on this important matter. It will be important to prospectively investigate pregnant women and their fetuses/neonates who are exposed to acetaminophen with inclusion of as many covariates as feasible.

We would like to conclude that at this moment there is no reason to discontinue the use of acetaminophen in pregnant women and their neonates because the alternative, the use of opioids, has proven to result in a dramatic increase in opioid-addicted neonates across many countries worldwide, while exposure to other nonsteroidal anti-inflammatory drugs like ibuprofen or indomethacin is associated with a much higher risk for fetal ductal closure when compared to acetaminophen [17]. 
Lastly, we would like to advocate using appropriate dosing of acetaminophen in pregnant women and their newborns as we still consider this as a "real" drug with effects and potential side effects $[18,19]$. This suggestion is in line with a recently revised summary of product characteristics (the leaflet) for acetaminophen by the European Medicine Agency. The adapted wording (italics) reads "Epidemiological studies on neurodevelopment in children exposed to paracetamol in utero show inconclusive results. If clinically needed, paracetamol can be used during pregnancy however it should be used at the lowest effective dose for the shortest possible time and at the lowest possible frequency" [20].

\section{Disclosure Statement}

The authors have no conflicts of interest to declare.

\section{Funding Sources}

None.

\section{Author Contributions}

Prof. Allegaert and Prof. van den Anker designed, wrote and reviewed several versions of this commentary.

\section{References}

1 Saugstad OD. Acetaminophen and the developing brain. Reason for concern? Neonatology. DOI: 10.1159/000505954.

2 Starko KM, Ray CG, Dominguez LB, Stromberg WL, Woodall DF. Reye's syndrome and salicylate use. Pediatrics. 1980 Dec;66(6): 859-64.

3 American Psychiatric Association. Diagnostic and Statistical Manual of Mental Disorders. 5th ed. Arlington, VA, USA: American Publishing; 2013.

4 Saunders A, Woodland J, Gander S. A comparison of prenatal exposures in children with and without a diagnosis of autism spectrum disorder. Cureus. 2019 Jul;11(7):e5223.

5 Schultz ST. Can autism be triggered by acetaminophen activation of the endocannabinoid system? Acta Neurobiol Exp (Wars). 2010;70(2):227-31.

6 Philippot G, Gordh T, Fredriksson A, Viberg $\mathrm{H}$. Adult neurobehavioral alterations in male and female mice following developmental exposure to paracetamol (acetaminophen): characterization of a critical period. J Appl Toxicol. 2017 Oct;37(10):1174-81.

7 Philippot G, Hallgren S, Gordh T, Fredriksson A, Fredriksson R, Viberg H. A cannabinoid receptor type 1 (CB1R) agonist enhances the developmental neurotoxicity of acetaminophen (paracetamol). Toxicol Sci. 2018 Nov; 166(1):203-12.

8 Philippot G, Nyberg F, Gordh T, Fredriksson A, Viberg H. Short-term exposure and longterm consequences of neonatal exposure to $\Delta$ (9)-tetrahydrocannabinol (THC) and ibuprofen in mice. Behav Brain Res. 2016 Jul;307: $137-44$.
9 Masarwa R, Levine H, Gorelik E, Reif S, Perlman A, Matok I. Prenatal exposure to acetaminophen and risk for attention deficit hyperactivity disorder and autistic spectrum disorder: a systematic review, meta-analysis, and meta-regression analysis of cohort studies. Am J Epidemiol. 2018 Aug;187(8):181727.

10 Damkier P. Prenatal exposure to acetaminophen and risk for attention deficit hyperactivity disorder and autistic spectrum disorder: a systematic review, meta-analysis, and metaregression analysis of cohort studies. Am J Epidemiol. 2018 Dec;187(12):2717-8.

11 Damkier $\mathrm{P}$, Pottegård $\mathrm{A}$, dePont Christensen R, Hallas J. Annotations and reflections: pregnancy and paracetamol: methodological considerations on the study of associations between in utero exposure to drugs and childhood neurodevelopment. Basic Clin Pharmacol Toxicol. 2015 Jan;116(1):2-5.

12 Olsen J, Liew Z. Fetal programming of mental health by acetaminophen? Response to the SMFM statement: prenatal acetaminophen use and ADHD. Expert Opin Drug Saf. 2017 Dec;16(12):1395-8.

13 Bittker SS, Bell KR. Postnatal acetaminophen and potential risk of autism spectrum disorder among males. Behav Sci (Basel). 2020 Jan 1;10(1).pii: E26.

14 Ji Y, Azuine RE, Zhang Y, Hou W, Hong X, Wang G, et al. Association of cord plasma biomarkers of in utero acetaminophen exposure with risk of attention-deficit/hyperactivity disorder and autism spectrum disorder in childhood. JAMA Psychiatry. 2019 Oct 30: $1-11$.
15 Alberti A, Pirrone P, Elia M, Waring RH, Romano C. Sulphation deficit in "low-functioning" autistic children: a pilot study. Biol Psychiatry. 1999 Aug;46(3):420-4.

16 Ystrom E, Gustavson K, Brandlistuen RE, Knudsen GP, Magnus P, Susser E, et al. Prenatal exposure to acetaminophen and risk of ADHD. Pediatrics. 2017 Nov; 140(5): e20163840.

17 Allegaert K, Mian P, Lapillonne A, van den Anker JN. Maternal paracetamol intake and fetal ductus arteriosus constriction or closure: a case series analysis. Br J Clin Pharmacol. 2019 Jan;85(1):245-51.

18 Allegaert K, van den Anker JN. Perinatal and neonatal use of paracetamol for pain relief. Semin Fetal Neonatal Med. 2017 Oct;22(5): 308-13.

19 Mian P, Allegaert K, Conings S, Annaert P, Tibboel D, Pfister M, et al. Integration of placental transfer in a fetal-maternal physiologically based pharmacokinetic model to characterize acetaminophen exposure and metabolic clearance in the fetus. Clin Pharmacokinet. 2020 Feb; Epub ahead of print. https://doi. org/10.1007/s40262-020-00861-7.

20 European Medicines Agency [cited from February 26th, 2020]. Available from: https:// www.ema.europa.eu/en/documents/pracrecommendation/prac-recommendationssignals-adopted-12-15-march-2019-pracmeeting_en.pdf. 